\title{
Observations of Pc 1-2 Waves in the Outer Magnetosphere
}

\author{
Stanley M. Kaye and Margaret G. Kivelson \\ Department of Earth and Space Sciences and Institute of Geophysics and Planetary Physics, University of California \\ Los Angeles, California 90024
}

\begin{abstract}
High time resolution measurements made inside the magnetopause from $L=7$ to $L=14$ by the University of California at Los Angeles fluxgate magnetometer aboard the Ogo 5 satellite demonstrate that Pc l-2 magnetic field oscillations are occasionally present in portions of the outer magnetosphere. Eleven events have been studied so far, and all of them occurred in the afternoon sector at geomagnetic latitudes from $0^{\circ}$ to $\pm 30^{\circ}$. Both the solar wind dynamic pressure and the magnetospheric cold plasma density were enhanced during events for which the respective data were available. The IMF had a southward component approximately 2 hours before the events; however, there was no significant correlation between the time of the events and either storm recovery phase or substorm onset. The magnetic field perturbations in each event were primarily transverse to the ambient field with amplitudes ranging from 2 to $8 \gamma$; however, small compressional oscillations, evidenced by 1 - to $2-\gamma$ fluctuations in the total field magnitude, were sometimes present. Most of the transverse perturbations exhibited lefthanded polarization. Brief excursions into the magnetosheath during two of the events revealed a highly disturbed magnetic field with significant power in frequencies of $\geq 0.1 \mathrm{~Hz}$. Although the magnetosheath was a source of free energy for waves at Pc 1-2 frequencies, the data within the magnetosphere suggest that the observed pulsations were ion cyclotron waves generated at the geomagnetic equator at large radial distances.
\end{abstract}

\section{INTRODUCTION}

The precipitation of ring current ions during the recovery phase of geomagnetic storms is an important mechanism for energy transfer from the magnetosphere to the ionosphere. The ions, energized as they adiabatically drift under the influence of a storm time enhanced convection electric field, may interact with electromagnetic waves with frequencies near the ion gyrofrequency in regions of sufficiently high cold plasma density [Cornwall, 1965; Kennel and Petschek, 1966]. An anisotropic energetic ion distribution peaked at large pitch angles can cause ion cyclotron wave growth. The ions will scatter toward lower energies and smaller pitch angles [Gendrin. 1968]; once inside the loss cone, the particles may precipitate.

Pc 1-2 (0.5-10 s) magnetic field oscillations are believed to be the magnetic signatures of low-frequency ion cyclotron waves generated in space near the geomagnetic equator. Ground studies (see reviews by Troitskaya and Gul'elmi [1967] and Gendrin [1970, 1975]) have revealed two classes of structured Pc l's. Pearls, which display characteristics of a wave packet bounding along a field line, are thought to be caused by ion cyclotron resonance occurring as the plasmaphere expands outward into the ring current during the recovery phase of geomagnetic storms [Cornwall et al., 1970; Russell and Thorne, 1970]. IPDP's (intervals of pulsation of diminishing period) result when substorm-injected ions encounter either the plasmasphere or enchanced cold plasma density regions [Solomon, 1975; Lin and Parks, 1976]. Roth and Orr [1975] have concluded that the Pc 1 source region lies close to the plasmapause. Their study compared the latitude of the largest magnetic fluctuations along a latitudinal chain of four ground stations with the nighttime plasmapause position determined from an empirical fit to the $K p$ dependence of the Chappell et al. [1970] equatorial plasma data. The ground stations used in their study, however, did not extend to geomagnetic latitudes greater than $67^{\circ}(L \simeq 6.6)$. Heacock [1974] and Feigin et al. [1978] report observations of Pc l's near $L=8$ on the dayside, far removed from the plasmapause. Both studies suggested that the wave observations were ion cyclotron waves generated

Copyright $\odot 1979$ by the American Geophysical Union. as hot protons drifted into regions of enhanced cold plasma density on the dayside. Feigin et al. [1978] theoretically determined that the growth rate of ion cyclotron waves generated near $L=8-9$ on the dayside would maximize for a cold particle density of $\sim 40 / \mathrm{cm}^{\mathrm{a}}$.

The difficulty in determining the Pc 1 source location from ground observations is complicated by the effects of ionospheric conductivity. Currents induced in the $E$ and $F$ layers by an incident wave can attenuate, duct, and change the polarization of the transmitted signal [Greifinger, 1972; Greifinger and Greifinger, 1973]. Studies of Pc 1 pulsations in space, which are essential in determining the wave generation region, are relatively sparse and, until this year, were based only on data from geosynchronous satellites [Dwarkin et al., 1971; McPherron et al., 1972; McPherron. 1978; Bossen et al., 1976a, b]. More recently, Geos 1 has explored the region earthward of its 6.7$\boldsymbol{R}_{E}$ apogee. Preliminary results of the on-board ULF experiment indicate that Pc 1 events are mostly seen near geosynchronous orbit, from $L=6.5$ to $L=7.1$ [ $S$-300 Experimenters, 1978].

Bossen et al. [1976b] correlated observations of Pc 1's in space and on the ground by taking simultaneous wave measurements aboard the ATS 1 satellite and at two nearly conjugate Canadian ground stations, Tungsten, Northwest Territories, and Ralston, Alberta. Although the ground signals were classified as IPDP's, the period of the waves observed aboard ATS 1 was constant. On the basis of these results the authors suggested that the Pc 1 source region is extended in space, and, whereas a satellite is capable of making only a single point measurement, the ground stations sample waves from a large spatial area.

Although they are capable of sampling distinct local time regions, geosynchronous satellites are nevertheless restricted to a very small range of latitudes and radial distances. A satellite with a highly elliptic orbit, however, is able to contribute to our understanding of Pc 1-2 wave generation in the magnetosphere by sampling distinct spatial regions over a large range of radial distances. Geos 1 will be useful for exploring regions inside $L=7.1$. We are able to examine Pc 1 2 waves outside $L=7$ using data from the University of 


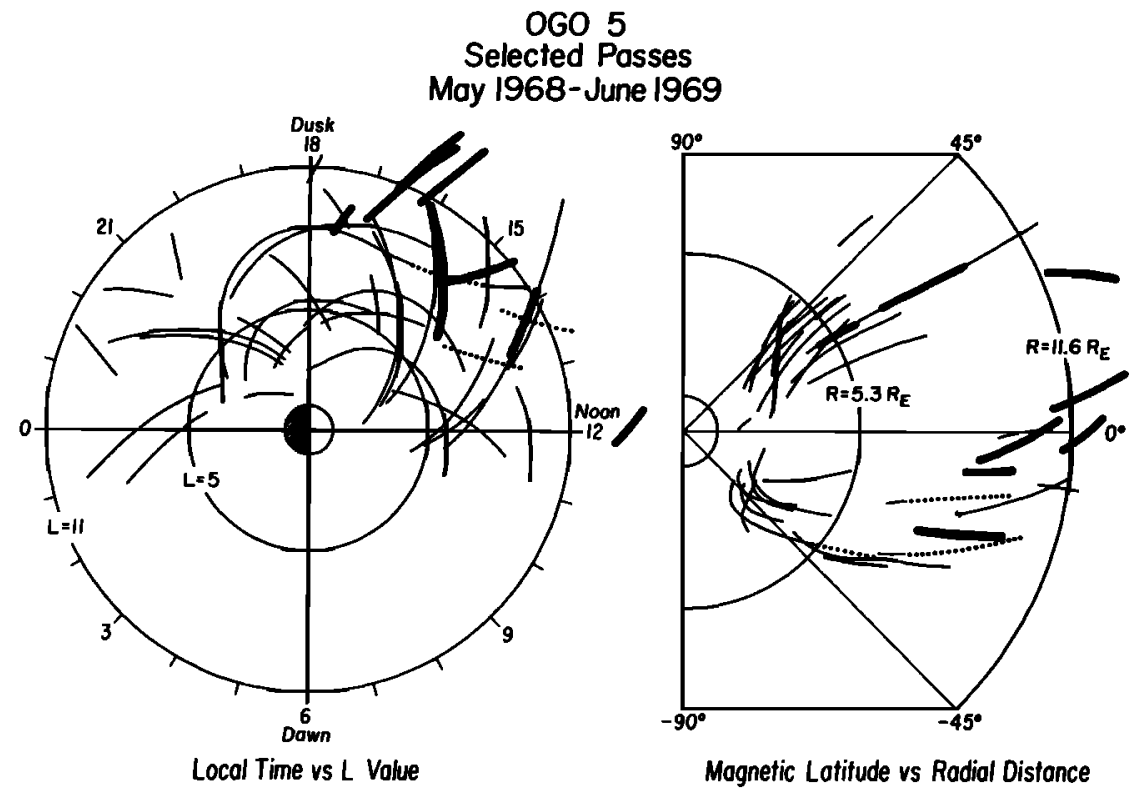

Fig. 1. Coverage of Ogo 5 between March 1968 and June 1969 for time periods selected by method A (light solid lines), events found by method A (dotted lines), and events found by method B (heavy solid lines). Methods A and B are described in the text.

California at Los Angeles (UCLA) triaxial fluxgate magnetometer aboard the highly elliptic Ogo 5 satellite. Two and one-half years of usable high temporal resolution data are available for the study. The Ogo 5 magnetometer has a noise level of $\simeq 0.1 \gamma$ at $10^{-1} \mathrm{~Hz}$, a digital window of $1 \gamma$, and high temporal resolution data sampling rates of $\geq 1$ sample per $0.144 \mathrm{~s}$ [Aubry et al., 1971], making it an almost ideal instrument with which to study Pc 1-2 magnetic field oscillations in the outer magnetosphere.

Using the Ogo 5 data, we present here the results of the first systematic search for Pc 1-2 magnetic field pulsations beyond synchronous orbit, from $L=7$ to $L=14$. We have identified 11 events within the time period from March 1968 to February 1969. We define an event as a portion of one day during which Pc 1-2-oscillations occurred. All of the events occurred in the afternoon quadrant of the magnetosphere. All were associated with both enhanced pressure regions in the solar wind and a southward component of the interplanetary magnetic field (IMF). In all cases for which data were available the magnetospheric cold $\mathrm{H}^{+}$ion density was seen to exceed the normal trough density. The pulsations themselves were primarily transverse to the ambient field, and the polarization of the waves was predominately left handed for most of the events.

Based on a comparison between instrument sensitivity and Pc 1-2 observations at various spacecraft, we consider the observed relations between our events and interplanetary conditions, as well as direct comparisons between our results and those at ATS 1, ATS 6, and Geos 1, to be meaningful. The noise levels of the instruments aboard the three mentioned spacecraft are $0.09 \gamma, 0.06 \gamma$, and $0.03 \gamma$, respectively [R. L. McPherron, unpublished manuscript, 1976; S-300 Experimenters, 1978]; however, the frequency of Pc 1 observations at each satellite is approximately equal, being 1 event per 2-3 days [Bossen et al., 1976a; R. L. McPherron, private communication, 1978; S-300 Experimenters, 1978]. The similarity of the $\mathrm{Pc} 1$ observation frequencies suggests that wave amplitudes were well above the instrument noise levels, rendering differences in instrument sensitivity unimportant. The sensitivity of the Ogo 5 magnetometer at $10^{-1} \mathrm{~Hz}$ is essentially the same as that aboard ATS 1, and, as will be seen, the amplitudes of our observed Pc 1-2 waves were also large compared with instrument noise levels. We therefore feel that few events were missed because of magnetometer sensitivity.

In the following sections we discuss the approach to the data selection, the solar wind and geomagnetic conditions at the time of the events, and the data themselves, and we conclude with a summary and discussion in which we suggest that we have observed ion cyclotron waves which were generated at radial distances well beyond the plasmapause.

\section{Selection Criteria}

The time periods for which the high-resolution magnetic field data were to be processed were chosen in two ways. The primary objective of the first approach was to determine whether Pc 1-2 pulsations could be found in the outer magnetosphere. Accordingly, the initial search was focused on portions of the data which satisfied conditions found to maximize the probability of Pc 1-2 observations at synchronous orbit [Bossen et al., 1976a]. Times were chosen in which Ogo 5 was in the afternoon-evening sector of the magnetosphere either during the recovery phase of a geomagnetic storm, as determined by $D s t$, or within hours after the onset of a substorm expansion phase as determined by mid-latitude magnetograms and $A E$. Using the above guidelines, which we shall call method $A$, a total of 45 hours on 30 different days were studied. We found events on 3 different days, with pulsations occurring for a total duration of 2 hours, $50 \mathrm{~min}$.

Having established the existence of Pc 1-2 pulsations beyond $L=7$ by method $A$, we used a second search method to establish the spatial distribution of the events. In this approach, method B, 4.6-s-averaged magnetic field data plots were scanned by eye. Plots of these data for 7 months, from March to June 1968 and from September 1968 to January 1969 , were available. Most local time sectors were covered by the Ogo 5 orbit during these intervals. The 4.6-s averages of the magnetic field data limit the frequency range for which events can be observed; only waves whose periods were greater than $9.2 \mathrm{~s}$ could be seen, although aliased power produced by 
fluctuations with periods down to $\sim 5 \mathrm{~s}$ was sometimes discerned.

Because method B does not identify events at the highfrequency end of the Pc 1-2 band, there are some limitations on its usefulness. The spatial region over which it should enable us to identify events can be estimated by noting that observed Pc 1 frequencies at synchronous orbit are typically $10-20 \%$ of the proton gyrofrequency at the geomagnetic equator [Bossen et al., 1976a]. Assuming that this is true for the entire magnetosphere and that the earth's magnetic field is essentially that of a dipole, method B should identify Pc 1-2 pulsations beyond $L \simeq 7.5$.

A total of 1364.5 hours of data, representing all the times within the 7 months that Ogo 5 was in the magnetosphere, was examined using method $B$, and events lasting for an additional 8 hours, 5 min on 8 different days were found. It is possible that the frequency limitations of method $B$ affected these results by failing to identify some high-frequency events. The event found nearest the earth was at $L=7.1$, in agreement with the above estimate of the spatial extent over which method B is useful.

In Figure 1 we show the coverage of Ogo 5 for the time periods studies by method A (light solid lines), the events found by method $\mathrm{A}$ (dotted lines), and the events found by method B (heavy solid lines). All of the events found fall in the noon-dusk quadrant despite equivalent satellite coverage in the dusk-midnight quadrant with method $A$ and in all quadrants with method $B$. As is demonstrated in the righthand panel of Figure 1 , the events are not confined to any specific geomagnetic latitude. A list of the dates and times of the events is given in Table 1 . Note that the events are subdivided into intervals of 20-30 min for purposes of spectral analysis. The length of each data interval was chosen on the basis of the sampling rate of the magnetometer at that time and the total duration of the event. Also given in Table 1 is the satellite position midway through each data interval, the period and amplitude of the peak spectral component determined by a Fourier analysis of the magnetic field data in that interval [McPherron et al., 1972], and the method by which the event was found. Additional 4.6-s-average data through February 1969 became available after the completion of this study, and two of the three events identified by method $A$ were seen in the new data. The higher-frequency event on February 5 , 1969, could not be identified by method $B$.

The spatial region within or near the dusk plasmapause is a likely ion cyclotron wave generation region [Cornwall, 1977]; however, our ability to observe Pc 1-2 oscillations at these low $L$ values was inhibited by two factors. In high field regions,

TABLE 1. List of Pc 1-2 Events

\begin{tabular}{|c|c|c|c|c|c|c|c|}
\hline Date & Universal Time & $L$ & $\lambda_{m}$ & $\begin{array}{l}\text { Local } \\
\text { Time }\end{array}$ & $\begin{array}{l}\text { Wave } \\
\text { Period }\end{array}$ & $\begin{array}{c}\text { Average } \\
\text { Peak-Peak } \\
\text { Transverse } \\
\text { Ampli- } \\
\text { tude, } \gamma\end{array}$ & $\begin{array}{l}\text { Method } \\
\text { by } \\
\text { Which } \\
\text { Found }\end{array}$ \\
\hline \multirow[t]{4}{*}{ Oct. 9,1968} & $0320-0340$ & 7.1 & 31.8 & 1437 & 5.1 & 0.9 & B \\
\hline & $0340-0400$ & 8.0 & 31.8 & 1502 & 4.9 & 1.0 & \\
\hline & $0420-0440$ & 9.5 & 30.9 & 1540 & 9.2 & 0.6 & \\
\hline & $0440-0500$ & 10.1 & 30.2 & 1556 & 10.3 & 3.3 & \\
\hline \multirow[t]{3}{*}{ Nov. 9,1968} & 0920-0945 & 9.7 & 31.4 & 1334 & 3.7 & 0.6 & B \\
\hline & $1000-1020$ & 11.0 & 30.8 & 1401 & 7.7 & 2.1 & \\
\hline & $1020-1040$ & 11.6 & 30.6 & 1412 & 10.3 & 2.8 & \\
\hline \multirow[t]{2}{*}{ Dec. 7,1968} & $1700-1720$ & 9.0 & -7.4 & 1723 & 11.5 & 1.4 & B \\
\hline & $1720-1735$ & 8.6 & -8.0 & 1729 & 12.5 & 1.1 & \\
\hline \multirow[t]{4}{*}{ Dec. 12,1968} & $1902-1922$ & 12.6 & 6.5 & 1624 & 15.4 & 1.4 & B \\
\hline & $1922-1942$ & 12.2 & 6.1 & 1627 & $\begin{array}{r}13.2 \\
7.1\end{array}$ & $\begin{array}{l}1.6 \\
1.1\end{array}$ & \\
\hline & $1942-2002$ & 11.8 & 5.7 & 1631 & & 1.4 & \\
\hline & $2002-2022$ & 11.4 & 5.1 & 1634 & $\begin{array}{l}6.2 \\
5.4\end{array}$ & $\begin{array}{l}0.9 \\
0.5\end{array}$ & \\
\hline \multirow[t]{5}{*}{ Dec. 15,1968} & $1150-1210$ & 10.5 & -18.6 & 1640 & 11.5 & 2.6 & B \\
\hline & $1210-1230$ & 10.1 & -19.3 & 1646 & 11.5 & 0.9 & \\
\hline & $1230-1250$ & 9.7 & -20.0 & 1652 & 11.5 & 0.7 & \\
\hline & & & & & 8.4 & 0.8 & \\
\hline & $1250-1310$ & 9.2 & -20.9 & 1700 & 8.4 & 0.7 & \\
\hline Dec. 18,1968 & $0058-0123$ & 11.1 & -1.7 & 1612 & 12.6 & 2.2 & B \\
\hline \multirow{2}{*}{ Dec. 21,1968} & $0320-0350$ & 13.7 & 23.7 & 1201 & 17.0 & 1.3 & B \\
\hline & $0350-0420$ & 14.2 & 22.8 & 1209 & 13.6 & 2.0 & \\
\hline \multirow[t]{4}{*}{ Jan. 7, 1969} & $2120-2140$ & 10.0 & -0.3 & 1450 & 11.5 & 3.6 & B \\
\hline & $2140-2200$ & 9.6 & -1.7 & 1456 & 9.2 & 4.5 & \\
\hline & $2200-2220$ & 9.2 & -3.3 & 1502 & 9.2 & 1.8 & \\
\hline & $2220-2240$ & 8.7 & -5.1 & 1508 & 9.2 & 1.6 & \\
\hline \multirow[t]{2}{*}{ Jan. 18, 1969} & $0930-0955$ & 8.8 & -33.8 & 1509 & 8.6 & 2.1 & $\mathbf{A}$ \\
\hline & $0955-1020$ & 8.4 & -37.1 & 1529 & 8.6 & 1.7 & \\
\hline \multirow[t]{3}{*}{ Jan. 26, 1969} & $0300-0320$ & 10.3 & -21.8 & 1347 & 5.4 & 3.3 & A \\
\hline & $0400-0420$ & 9.6 & -26.8 & 1406 & 6.8 & 1.1 & \\
\hline & $0420-0440$ & 9.4 & -28.5 & 1413 & 5.3 & 1.2 & \\
\hline \multirow[t]{3}{*}{ Feb. 5, 1969} & $1313-1333$ & 9.0 & -12.8 & 1312 & 2.0 & 2.1 & A \\
\hline & $1333-1353$ & 8.5 & -13.5 & 1319 & 1.7 & 1.7 & \\
\hline & $1353-1413$ & 8.1 & -4.7 & 1329 & 1.6 & 1.0 & \\
\hline
\end{tabular}

Given are the days and times of the intervals, satellite position midway through each data interval, wave period, average transverse peak-peak amplitude of oscillation, and method by which the event was found. 


\section{SOLAR WIND PARAMETERS}
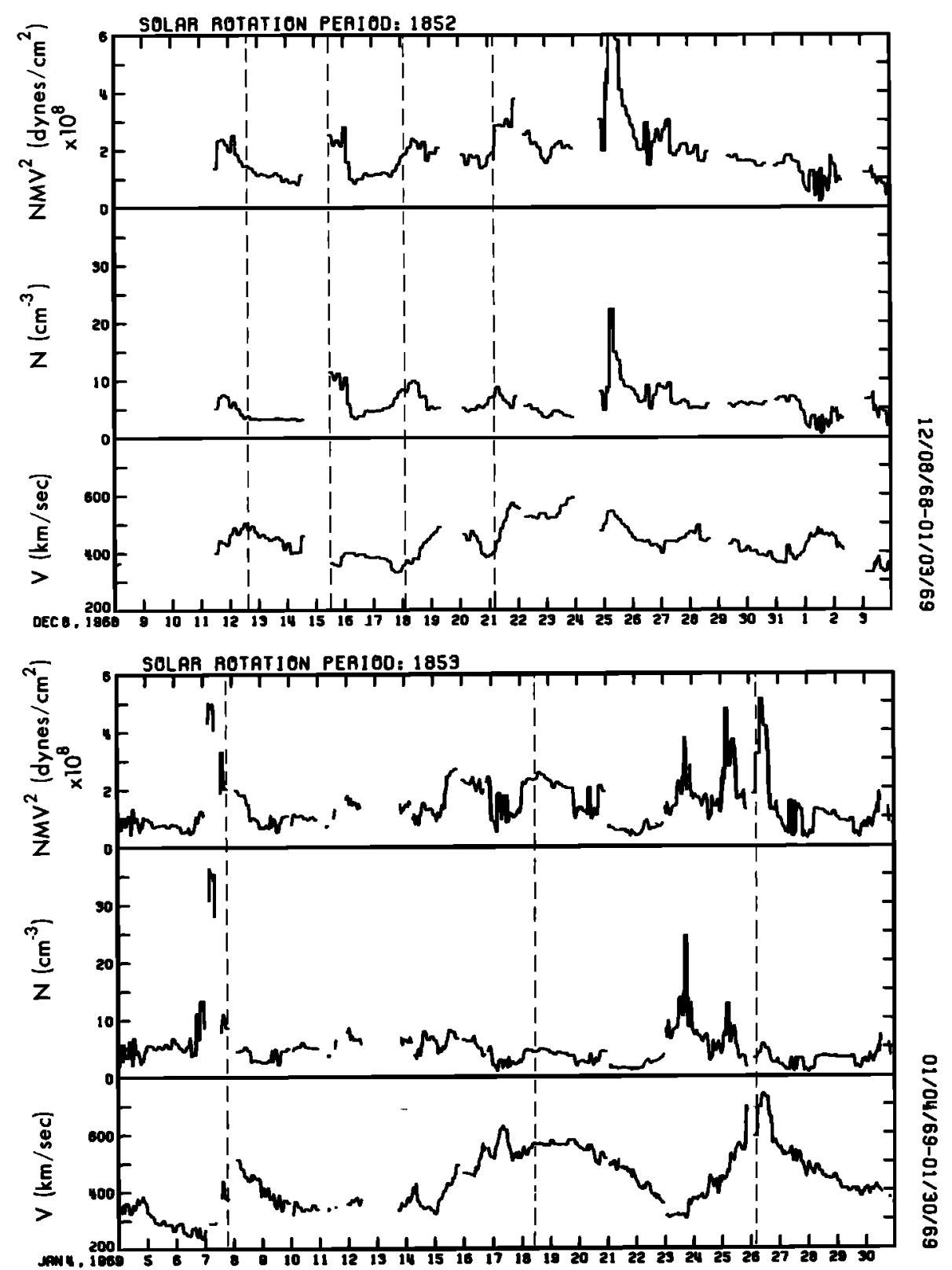

Fig. 2. Solar wind velocity, density, and dynamic pressure for two solar rotations during which seven events occurred. The vertical dashed lines denote the times of the seven events.

wave frequencies at $10-20 \%$ of the proton gyrofrequency are too high to be identified by method $B$. In addition, apparent field oscillations caused by the vibrating magnetometer boom aboard Ogo 5 were consistently seen inside $5 R_{E}$ and may have obscured the presence of natural waves. The amplitude of the artificial oscillation reached 5-10 $\gamma$ in the region of interest, and the frequency of oscillation was in the high-frequency end of the Pc I band (2-5 s). Although we were not capable of detecting Pc 1 signals at low $L$, preliminary results from $G e o s$ 1 [S-300 Experimenters, 1978] and previous studies of higherfrequency $(1-56.5 \mathrm{~Hz})$ waves [Taylor et al., 1975; Taylor and Lyons, 1976; Kintner and Gurnett, 1977] have shown that ion cyclotron waves are an infrequent phenomenon at low $L$.

\section{Solar Wind Conditions and Geomagnetic Activity}

In Figure 2 we show solar wind velocity, density, and dynamic pressure for solar rotations 1852 and 1853 , two solar rotations within which 7 of the 11 Pc 1-2 events occurred. The vertical dashed lines denote the times of the events. Each of the events is clearly associated with a region of enhanced solar wind dynamic pressure. To be even more specific, 6 of the 7 events shown occurred within a few hours of the time when the leading edge of the enhanced pressure region passed the earth. Only 1 of the 7 events shown, December 12, was not associated with the leading edge of the region. For this event, Ogo 5 was not in the magnetosphere at the time that the leading edge passed the earth, but later, when Ogo entered the magnetosphere, oscillations were present. Solar wind data exist for only one additional event not shown in the figure. The event occurred on February 5, 1969, and was also concurrent with the arrival at earth of the leading edge of a high-pressure region.

To establish a more quantitative relation between enhanced solar wind pressure regions and Pc 1-2 events, we have exam- 


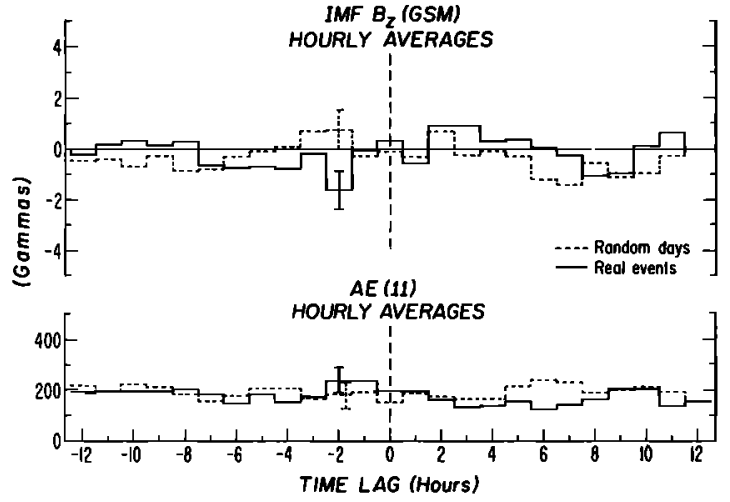

Fig. 3. Results of superposed epoch analyses of hourly averages of the $z$ component of the IMF in GSM coordinates and of AE. The analyses were performed for 12 hours on either side of the event (the time of the event is indicated by a vertical dashed line at zero time lag). The analyses were performed both for the events (solid lines) and for 11 times chosen at random (dashed lines). The error bars represent the standard deviation of the mean for each respective variable.

ined solar wind data, when available, for the 7 months covered by the method B search. We hoped to answer two specific questions. First, what is the probability of observing Pc 1-2 oscillations at the time that an enhanced solar wind pressure region passes the earth, and, second, is the afternoon quadrant a preferential region of Pc 1-2 wave activity? Recognizing the need to quantify what is meant by an enhanced pressure region, we used the results of Holzer and Slavin [1978] to define a threshold above which the solar wind pressure is to be considered enhanced. These authors determined the solar wind pressure distribution at the times of Ogo 5 magnetopause crossings for the years 1968-1970. Although a partial distribution is given in the Holzer and Slavin [1978] paper, the total distribution was approximately Gaussian with a mean solar wind pressure of $1.66 \times 10^{-8} \mathrm{dyn} / \mathrm{cm}^{2}$ and a standard deviation of $\sim$ $70 \%$ of the mean (J. Slavin, private communication, 1978). Using the value of one standard deviation greater than the mean, we define an enhanced pressure region as an interval during which the solar wind pressure exceeds $2.80 \times 10^{-8} \mathrm{dyn} /$ $\mathrm{cm}^{2}$.
For times when data were analyzed by method B, there were nine periods of enhanced solar wind pressure. We separate these nine periods into two groups, those for which Ogo was in the afternoon sector, to be called 'afternoon periods,' and those for which Ogo was near dawn, to be called 'morning periods.' The nine periods of enhanced solar wind pressure corresponded to five afternoon periods and four morning periods. Pc 1-2 events were found during or within a few hours of all five of the afternoon periods, but no events were found during the four morning periods. In addition, no Pc 1-2 event was found during extended intervals when the solar wind pressure remained less than $2.80 \times 10^{-8} \mathrm{dyn} / \mathrm{cm}^{2}$.

Although the statistics of the survey are quite limited, the results suggest that $\mathrm{Pc} 1-2$ events are preferentially seen in the afternoon sector and that they are associated with enhanced solar wind pressure on the magnetosphere. We cannot determine whether the existence of the Pc 1-2 waves was influenced by the magnitude of the solar wind pressure, the increase in pressure, or both. Sharp leading edges were characteristic of all five afternoon periods, but since Ogo was in the magnetosphere for only a portion of each period, we could not ascertain the precise relation between the onset of $\mathrm{Pc} 1-2$ oscillations and the time that the leading edge passed the earth. We do note that while the December 12 event was not associated with an increase in pressure, it was seen within a few hours after an interval of enhanced pressure. The afternoon quadrant has also been shown to be a preferential region for Pc 1-2 wave activity by Bossen et al. [1976a] and McPherron [1978]. R. L. McPherron (private communication, 1978) further showed an association between Pcl's seen at synchronous orbit and times at which the leading edge of an enhanced solar wind pressure region passed the earth.

To determine the relation of the time of the events to both the orientation of the IMF and substorm activity, superposed epoch analyses were performed using hourly averages of the north-south component of the IMF in GSM coordinates and hourly averages of the $A E$ index. The analyses used data for 12 hours on either side of the events. The results are shown as solid lines in Figure 3. To estimate the 'base line' of $B_{z}$ and $A E$, the same analysis was performed centered on 11 times chosen

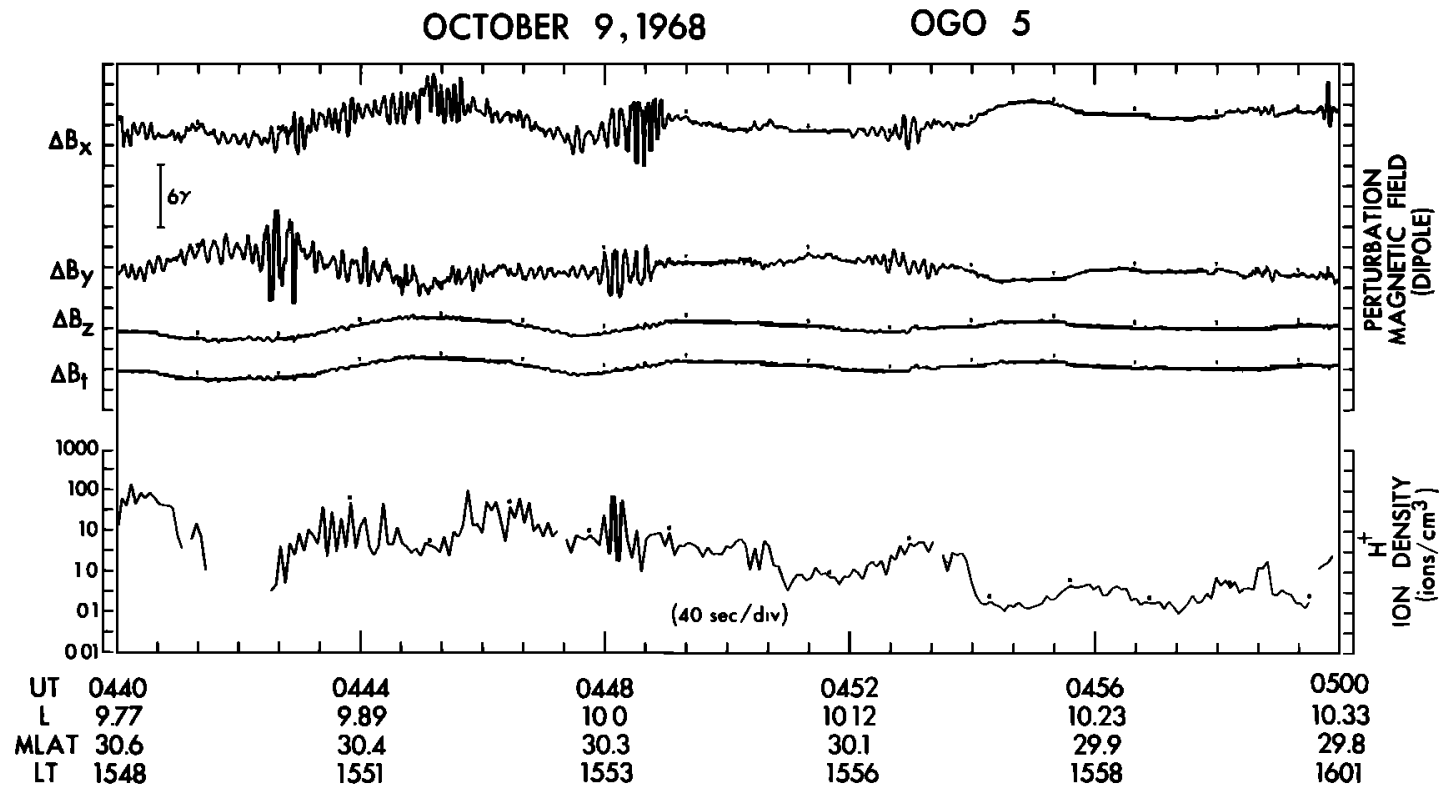

Fig. 4. Pc 1 event on October 9, 1968, from 0440 to 0500 UT. The perturbation magnetic field from high time resolution data (0.3-s averages) is plotted in dipole coordinates. Also shown is the $\mathrm{H}^{+}$ion density (10-s averages). The high-frequency oscillations in the ion density occurred at their Nyquist frequency and should be ignored. 
at random. The 11 random times contained both geomagnetically disturbed and quiet days. The results of these calculations are shown as dashed lines. The vertical dashed line at zero time lag denotes the time of the event, and each error bar is the calculated standard deviation of the mean of the respective variable. As can be seen from the figure, there is a significant southward component of the IMF approximately 2 hours prior to the event; there is, however, no nonrandom pattern for the hourly $A E$ index. Although we do not show the results here, the same type of analysis was performed using hourly averages of $A L$; as with $A E$, there is no relationship between the event occurrence and $A L$. In addition, the occurrence of the Pc 1-2 events does not show any dependence on the magnitude or variation of Dst.

\section{Data for Selected Pulsation Events}

October 9, 1968,0440-0500 UT. Perturbation magnetic field data (0.3-s averages) from the UCLA fluxgate magnetometer and 10-s-averaged $\mathrm{H}^{+}$ion density measured by the Lockheed light ion mass spectrometer are shown in Figure 4 for $20 \mathrm{~min}$ on October 9, 1968. The perturbation magnetic field is the measured magnetic field with the Jensen-Cain model internal field removed [Cain et al., 1967]. The field components are plotted in dipole coordinates in which $\hat{z}$ is along the model field line, $\hat{x}$ is radially out, and $\hat{y}$ is azimuthal, completing a right-hand coordinate system.

The satellite, at a position well off the geomagnetic equator at $\lambda_{m} \simeq 30^{\circ}, L \simeq 10$, and $1600 \mathrm{LT}$, recorded a Pc $1-2$ pulsation starting at $0320 \mathrm{UT}$ and continuing, intermittently, for 1.3 hours. Individual wave trains lasted for approximately 10-20 min. The oscillations occurred only in the transverse components of the magnetic field with a wave period of $\sim 10 \mathrm{~s}$ and an average peak-peak amplitude of $3.3 \gamma$. Both the magnetic field and the ion density were modulated by a small-amplitude long-period (2-3 min) disturbance during times of Pc 1-2 activity.

From the plot it can be seen that the Pc 1-2 pulsation activity maximized when the average ion density was large. During most of the event the average ion density was typically $5-30 / \mathrm{cm}^{3}$. At $0448: 20$ UT the ion density decreased to $\sim 1.0 /$

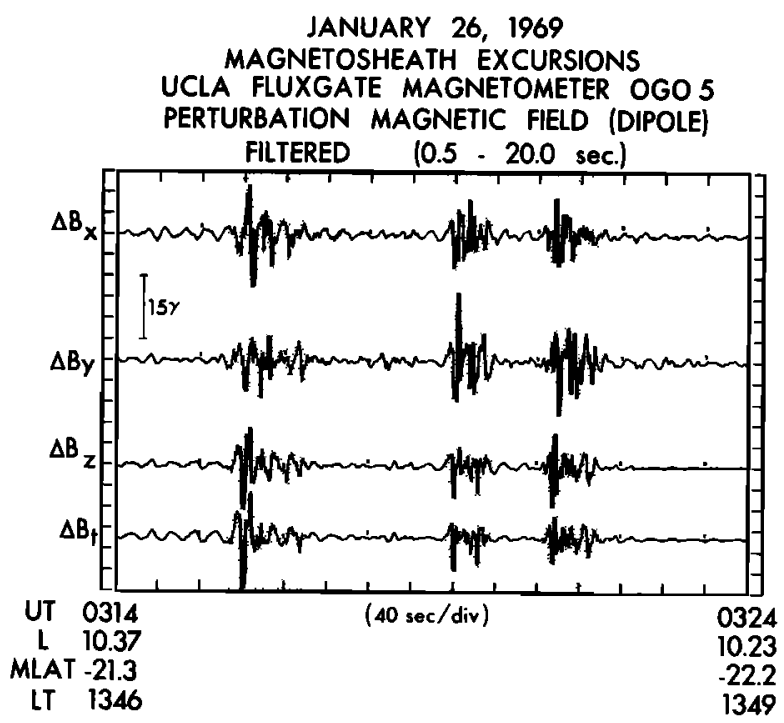

Fig. 5. Three magnetosheath crossings which occurred on January 26, 1969, from 0314 to 0324 UT. The field components are the same as those in Figures 5-8, and the shaded regions indicate periods when the satellite was in the magnetosheath. $\mathrm{cm}^{3}$, and the magnetic field perturbations ceased. The field oscillations grew again at 0452:00 UT as the ion density increased to $\sim 10 / \mathrm{cm}^{3}$ but quickly damped out as the density subsequently dropped to a value of $0.1-1.0 / \mathrm{cm}^{3}$. The correlation between periods of wave activity and periods of enhanced cold $\mathrm{H}^{+}$density was noted for all events for which the ion data exist.

It has been recognized for some time that the density values obtained from measurements of the Lockheed light ion mass spectrometer can be incorrect because the density was determined from the measurement of the ion flux along the spacecraft velocity vector [Kivelson, 1976; Kokubun et al., 1977]. The reported ion density used for the plots was obtained by dividing the flux measurement by the speed of the spacecraft [Harris and Sharp, 1969]. No correction was made for the possible motion of the ions themselves, although it is clear that a component of ion velocity directed into (away from) the detector would give rise to an overestimate (underestimate) of the density.

Plasma motions which can affect the density calculation result from the dawn-dusk convection electric field, electric fields of long-period hydromagmetic waves, and finite temperature of the plasma. For a cold plasma distribution modulated by a long-period wave, Kivelson [1976] showed that a good estimate of the actual density is the average reported density for each wave cycle. However, the reported densities may be off by a factor of 1-3 because of finite temperature effects (C. R. Chappell, private communication, 1976) and convection.

The average reported density, used here as a qualitative estimate of actual ion density, exhibited increases and decreases in Figure 4 which indicate that Ogo passed through spatial boundaries. The reported ion density values were not severely affected by the MHD disturbance which lasted from 0440 to $0448 \mathrm{UT}$, and the average density during this period was $\sim 10 / \mathrm{cm}^{3}$. The reported density dropped to $\sim 0.1 / \mathrm{cm}^{3}$ within a period of $20 \mathrm{~s}$ at 0454 UT. For a magnetic field strength of $50 \gamma$ and an ion temperature of $10 \mathrm{eV}$ we note that Ogo 5 , traveling at $6 \mathrm{~km} / \mathrm{s}$, can pass through a spatial boundary several thermal ion gyroradii thick in a few seconds. Time scales for changes in convective motions are considerably longer. We therefore conclude that the large reported ion densities seen during periods of Pc 1-2 wave activity actually represent regions of enhanced cold plasma density.

Although we have presented a case in which the oscillations were exclusively in the transverse components of the field, small $(\sim 1 \gamma)$ compressional components, which are identified as fluctuations in the total field, were seen in 6 of the 11 events at all geomagnetic latitudes and radial distances. When a compressional component was present in the magnetosphere, its amplitude was proportional to that of the transverse perturbation with a typical compressional to transverse amplitude ratio of $\sim 0.2$.

January 26, 1969, 0314-0324UT. Since all of our events occurred at large $L$ values, it was not unexpected that Ogo 5 would occasionally cross the magnetopause and enter the magnetosheath. These encounters afforded an opportunity to compare magnetic field fluctuations in the magnetosheath with the Pc 1-2 events in the magnetosphere. An example of three brief excursions into the magnetosheath within a span of $10 \mathrm{~min}$ on January 26, 1969, is shown in Figure 5. The three shaded regions represent times when the satellite was in the magnetosheath. The magnetosheath was identified by a marked increase of noise in all components of the magnetic field and by a sharp decrease of energetic particle fluxes as measured by the 


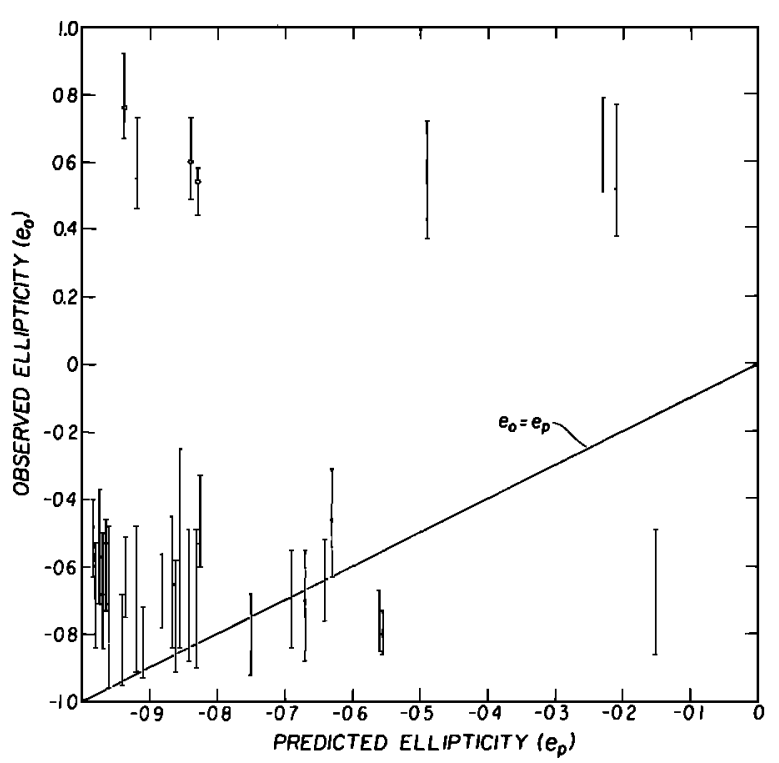

Fig. 6. Plot of observed ellipticity $e_{o}$ of the 32 Pc 1-2 events tabulated in Table $I$ versus the predicted ellipticity $e_{p} ; e_{p}$ was determined from (2) by the method described in the text. The vertical bars represent the range of observed ellipticity during each time interval. The open circles indicate that Ogo encountered the magnetosheath during or within $1 / 2$ hour of the time period in question.

Lawrence Livermore Laboratory energetic particle spectrometer. The orientation of the magnetic field was not used as a diagnostic, since often the field did not abruptly change direction across the magnetopause.

The magnetosheath field fluctuations shown in Figure 5 were similar to those in the magnetosphere in terms of amplitudes, 5-15 $\gamma$, and periods, 5-15 s. Other magnetosheath encounters on this day and on November 9, 1968, revealed oscillations with similar amplitudes and periods. The fluctuations in the magnetosheath and those in the magnetosphere differed in the magnitude of the compressional component. Whereas the ratio of compressional to transverse amplitude was $\sim 0.2$ in the magnetosphere, the ratio was $\sim 1.0$ in the magnetosheath. In addition, field fluctuations in the magnetosheath occurred at all frequencies within the Pc 1-2 band. As is seen in Figure 5, some longer-period wave activity of smaller amplitude was present just earthward of the magnetopause. These fluctuations in the magnetosphere suggest that some attenuated wave power was being transmitted from the magnetosheath into the magnetosphere, with the wave damping being greatest for the higher-frequency signals.

\section{SUMMARY AND DISCUSSION}

Eleven examples of Pc 1-2 magnetic field oscillations at large $L$ have so far been identified in the records of the Ogo 5 satellite. All of the events occurred in the noon-dusk quadrant of the magnetosphere and in regions of enhanced ambient cold $\mathrm{H}^{+}$ion density. Most of the events occurred within a few hours after the leading edge of an enhanced dynamic pressure region in the solar wind passed the earth. In addition, the IMF had a significant southward component approximately 2 hours prior to the events. The waves themselves were primarily transverse with large (1-8 $\gamma$ ) amplitudes. Brief excursions into the magnetosheath revealed 5- to $15-\gamma$ compressional oscillations in all components of the magnetic field at Pc 1-2 frequencies.

Bossen et al. [1976a, b], who observed Pc 1 signals at synchronous orbit on ATS 1, and Kintner and Gurnett [1977], Taylor et al. [1975], and Taylor and Lyons [1976], who ob- served higher-frequency signals within the plasmasphere aboard Hawkeye 1 and Explorer 45, respectively, attributed their observations to waves generated by ion cyclotron resonance. It is tempting to do the same here.

We must first ask what wave properties distinguish ion cyclotron waves from other modes of oscillation. One such property is the frequency of oscillation. Cold plasma theory allows the ion cyclotron wave frequency to assume any value below the ion gyrofrequency. However, for a temperature anisotropy in the resonant particle distribution with $T_{\perp} / T_{\|} \simeq$ 1.5 , the most easily destabilized ion cyclotron waves will have frequencies $\omega / \Omega^{+}=0.1-0.4$, where $\omega$ is the wave frequency and $\Omega^{+}$is the ion gyrofrequency at the generation region [Cornwall et al., 1970; Cornwall, 1977; Lin and Parks, 1976]. The Pc l observations at synchronous orbit exhibited frequencies with $\omega / \Omega_{p} \simeq 0.1-0.3$, where $\Omega_{p}$ was the local proton gyrofrequency [Bossen et al., 1976a, b; R. L. McPherron, private communication, 1978].

We have calculated the equatorial values of $\omega / \Omega_{p}$ for the events of this study using a dipole field model to transform the magnitude of the measured magnetic field to its value at the geomagnetic equator, the most probable ion cyclotron wave generation region. Assuming that the dominant ions were protons and that the wave frequency did not change as the wave propagated off the equator, we found that all but four of the data intervals contained waves with $\omega /\left.\Omega_{p}\right|_{\text {eq }}=0.1-0.4$. The three intervals on February 5, 1969, and the interval from 0920 to 0945 UT on November 9, 1968, contained waves with $\omega /\left.\Omega_{p}\right|_{\text {eq }}>0.4$.

A clear distinguishing feature of ion cyclotron waves is their polarization, which is left hand circular when the wave is guided along the magnetic field. In fact, we can readily compare the expected ellipticity of an ion cyclotron wave with the observed ellipticity. From Stix [1962] the expected ellipticity of an ion cyclotron wave, based on the cold plasma approximation and a uniform magnetic field, is given by the expression

$$
e_{p}=\frac{\sin ^{2} \Psi-\left(\sin ^{4} \Psi+4 x^{2} \cos ^{2} \Psi\right)^{1 / 2}}{2 x \cos \Psi}
$$

where $e_{p}$ is the predicted ellipticity, $\Psi$ is the angle between the wave normal vector and the magnetic field, and $x=\omega / \Omega_{p}$ (locally). For $x$ and $\Psi$ such that $4 x^{2} \cos ^{2} \Psi \gg \sin ^{4} \Psi$, that is, when the wave is guided along the magnetic field, the wave is left handed and nearly circular, since $e_{p} \simeq-1$. When $\psi$ is large, $e_{p} \simeq 0$, and the polarization is linear.

In order to compare the predicted ellipticity $e_{p}$ with the observed ellipticity $e_{o}$ the data in each interval were first band-

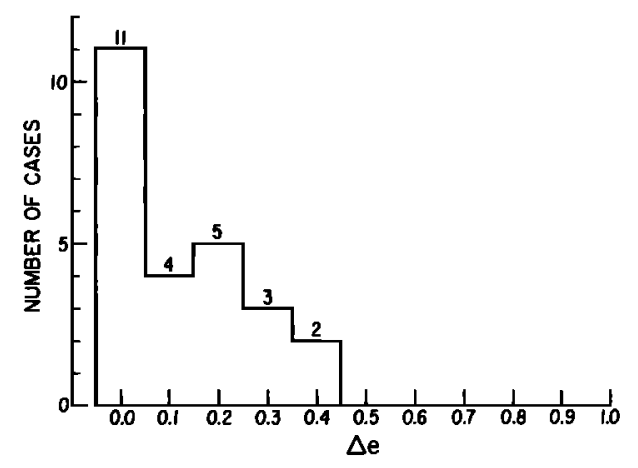

Fig. 7. Histogram of the number of cases in which the predicted ellipticity fell within $\Delta e$ of any portion of the range of observed ellipticity. $\Delta e$ is incremented by 0.1 . 
pass-filtered between 1 and $18 \mathrm{~s}$. Then $\Psi$ and $e_{o}$ were calculated for each interval using the coherency analysis techniques described by Fowler et al. [1967] and McPherron et al. [1972]. The sense of polarization was obtained from the phase relation between the transverse fluctuations in the principal axis system, with negative values of $e_{0}$ corresponding to left-handed polarization with respect to the magnetic field vector.

In Figure 6 we compare the observed ellipticity with the expected ellipticity. The open circles may be special cases, since Ogo encountered the magnetosheath during or within $\frac{1}{2}$ hour of the time period in question. The vertical bars indicate the range of ellipticities obtained for individual spectral components within the passband. Although the values of the observed ellipticities range from -0.96 to +0.63 , the predicted ellipticities are always negative for ion cyclotron waves.

If the open circle points from magnetosheath encounter days are ignored, the following features emerge from examination of Figure 6:

1. The majority of wave events exhibited left-handed ellipticity; right-handed polarization was observed for only 4 of 29 intervals.

2. Twenty-four of twenty-five points for which the magnitude of the predicted left-handed ellipticity was greater than 0.5 were left handed.

3. The magnitude of the predicted ellipticity exceeded 0.5 for only one of the right-handed points.

4. There was only one left-handed point with $\left|e_{p}\right|<0.5$.

To display the good agreement between $e_{p}$ and $e_{o}$ for the left-handed events, we have defined a quantity $\Delta e$ as the magnitude of the minimum difference between the predicted ellipticity and any point within the range of observed ellipticity. The histogram in Figure 7 shows the number of cases for which $\Delta e$ took on values between 0 and 1 . Of the 25 lefthanded events, 11 have observed ellipticity equal to the predicted ellipticity within the range of the observed ellipticity. Twenty of twenty-five have $\Delta e \leq 0.2$, and all of the observed values fall within $\Delta e=0.4$ of the predicted values.

Although there is not complete agreement between observation and theory, we can construct a scenario, based on our observations, consistent with the idea that most of the observed waves were generated by ion cyclotron instability of protons near the geomagnetic equator. In Figures 2 and 3 we showed evidence that the observed Pc 1-2 events occurred at the time that high-pressure regions in the solar wind passed the earth and a few hours after the IMF exhibited a significant southward component. A correlation between large northsouth components of the IMF and interaction regions in the solar wind was statistically proven by Rosenberg et al. [1978].

It is well established that enhanced magnetospheric convection is associated with an enhanced southward $B_{z}$ [Russell et al., 1974]. The solar wind electric field, directed dawn-dusk for the case of a southward $B_{z}$, is transmitted through the magnetopause boundary and is applied across the entire magnetosphere. Although this enhanced energy transfer from solar wind to magnetosphere sometimes results in explosive energy release in the tail, as in substorms, recent evidence has shown that the magnetosphere may attain a steady state at a higher energy level without substorms being triggered [Pytte et al., 1978]. We have shown that our events are not, in general, closely associated with either storms or substorms.

The enhanced cross-magnetospheric electric field will result in an enhanced transport of particles sunward. Cold plasma, initially corotating with the earth, will start to convect sunward, giving rise to ion density enhancements in the noon- dusk quadrant as seen on Ogo 5 [Chappell, 1974]. Energetic particles will also convect sunward; in particular, energetic ions will drift sunward through dusk and can develop a net pitch angle anisotropy from mechanisms such as charge exchange [Cornwall, 1977; Cowley, 1977] and pitch angle scattering from interactions with electrostatic waves [Ashour-Abdalla and Thorne, 1977]. Anisotropic ion distributions, upon encountering regions of enhanced plasma density, may cause left-hand elliptically polarized ion cyclotron waves with $\omega / \Omega_{p}$ $\simeq 0.1-0.4$ to grow. Although correlations between ELF noise and long-period ULF waves with enhanced density regions have been established in the outer magnetosphere [Kivelson, 1976], no correlation has previously been made between Pc 12 oscillations and enhanced density regions.

A second mechanism which can produce elliptically polarized waves near the magnetopause is the Kelvin-Helmholtz instability. Southwood [1968] and Chen and Hasegawa [1974a, $b$ ] have discussed the Kelvin-Helmholtz instability of the magnetopause boundary as a source for longer-period (Pc 3-5) pulsations, but this mechanism has not been ruled out as a source for higher-frequency pulsations. The polarization of these waves would be right handed earthward of the magnetopause in the noon-dusk quadrant and could account for the right-hand polarization of the three open circle points in Figure 6 obtained for Pc 1-2 intervals near the magnetopause. In the discussion of Figure 5 we suggested that some wave power was transmitted from the magnetosheath into the magnetosphere, and it is possible that the waves present in the magnetosphere during these time intervals were produced by a source at the magnetopause rather than internal magnetospheric processes.

As is seen in Figure 6, there are also four time intervals not closely associated with magnetosheath encounters which contain right-hand-polarized waves. Two possible explanations of the observed polarization have occurred to us. A source at the magnetopause cannot be ruled out, because there is no way of knowing just how close the spacecraft was to the magnetopause. Alternatively, we note that three of the four points have small predicted ellipticities which indicate that the wave is propagating at a large angle to the magnetic field. It is possible that ion cyclotron waves which are propagating at large angles with respect to the field, and therefore are nearly linearly polarized, could mode-convert into fast waves (J. Maggs, private communication, 1978). The fast waves could account for the occasional observation of right-handed ellipticity.

We can gain some insight into the extent of the wave generation region by comparing the Pc 1-2 occurrence frequency at Ogo 5 with the frequencies at ATS 1 and ATS 6. Since Ogo 5 spends most of its 2.5-day orbit in the solar wind, the probability of observing a Pc 1-2 event per orbit is smaller for Ogo than for ATS 1 and ATS 6, which nominally spend their entire 24-hour orbit in the magnetosphere. To eliminate, as much as possible, the possible consequences of the dissimilarity of the satellites' orbits, we define the occurrence frequency to be the number of Pc 1-2 events observed per examination hour. The number of examination hours is defined to be the time spent by the satellite in a region where it is able to observe $\mathrm{Pc} 1-2$ waves of magnetospheric origin. Geosynchronous satellites will have 24 examination hours per orbit. The number of examination hours for Ogo 5 is taken to be the total time spent in the magnetosphere from $L=7$ to $L=14$ irrespective of local time position. Using the times covered by the method $B$ search to calculate the total number of examination hours for Ogo 5 , we find the occurrence frequency to be $\sim 1 / 150$. In contrast, the 
occurrence frequency at both ATS 1 and ATS 6 is $\sim 1 / 60$ [Bossen et al., 1976a, b; R. L. McPherron, private communication, 1978]. Preliminary results indicate that the Pc 1-2 occurrence frequency at Geos 1 , which spends most of its 12-hour orbit near $6.6 R_{E}$, is comparable to that at geosynchronous orbit [S-300 Experimenters, 1978].

The occurrence frequency of Pc 1-2 waves is seen to be significantly lower beyond geosynchronous orbit than at or near it. Furthermore, the average event duration at ATS 1 was 15-30 min [Bossen et al., 1976a], while events lasted for $\geq 1$ hour at Ogo (see Table 1). One possible explanation of such results is that the $\mathrm{Pc} 1-2$ generation region is radially limited with an outer boundary which occasionally moves outside $L \simeq$ 7. Longer-duration events will be seen at Ogo if the generation region which extends to large $L$ is longer lived than that which terminates near the ATS orbit.

However, special assumptions regarding the shape of the generation region can equally well account for the observed occurrence frequencies. We note that whereas the ATS satellites followed azimuthal trajectories, Ogo's orbital motion through the outer magnetosphere was predominantly radial. If the Pc 1-2 generation region is radially extended but confined to a limited range of local times centered at an arbitrary local time in the postnoon quadrant, then ATS 1 and ATS 6, which covered all local times in one orbit, could conceivably observe a greater number of events than Ogo. The radially moving Ogo would see a longer-duration event if its orbit fortuitously coincided with the radially extended generation region. With the present set of measurements it is not possible to distinguish between the two suggested explanations.

In conclusion, we believe that most of our observed wave events suggest that ion cyclotron waves are being generated in the outer magnetosphere, consistent with ground observations by Heacock [1974] and Feigin et al. [1978]. In addition, the few intervals of right-hand-polarized waves seen in Figure 6 suggest that a second source of Pc 1-2 waves other than ion cyclotron resonance must exist. We were not able to determine the nature of this second mechanism in the present study. Nevertheless, our results indicate that the plasmapause region can no longer be regarded as the only generation region of ion cyclotron waves.

Acknowledgments. The authors wish to thank the National Space Science Data Center for providing the solar wind data and the energetic electron and proton data of $\mathrm{H}$. I. West, Jr. We wish also to thank J. D. Maggs for discussions concerning the propagation characteristics of Alfven waves, R. L. McPherron for discussions concerning his results at geosynchronous orbit, and $\mathbf{H}$. J. Singer for first pointing out that $\mathrm{Pc} 1-2$ events could be identified from the 4.6-s-average magnetic field data and helping to identify them. We would finally like to thank C. T. Russell, who, in addition to sharing his wealth of knowledge with us, was initially responsible for casting the Ogo 5 magnetometer results into an extremely workable data base. This work has been supported in part by NASA grants NSG-7295 and NGL 05-007-004 and by NSF grant DES 74-23464. UCLA Institute of Geophysics and Planetary Physics publication 1830.

The Editor thanks R. Gendrin and L. J. Lanzerotti for their assistance in evaluating this paper.

\section{REFERENCES}

Ashour-Abdalla, M., and R. M. Thorne, The importance of electrostatic ion cyclotron instability for quiet time proton auroral precipitation, Geophys. Res. Lett., 4, 45, 1977.

Aubry, M. P., M. G. Kivelson, and C. T. Russell, Motion and structure of the magnetopause, J. Geophys., 76, 1673, 1971.

Bossen, M., R. L. McPherron, and C. T. Russell, A statistical study of Pc 1 magnetic pulsations at synchronous orbit, J. Geophys. Res., 81, 6083, 1976a.

Bossen, M., R. L. McPherron, and C. T. Russell, Simultaneous Pc1 observations by the synchronous satellite ATS-1 and ground stations: Implications concerning IPDP generation mechanisms, $J$. Atmos. Terr. Phys., 38, 1157, 19766.

Cain, J. C., S. J. Hendricks, and R. A. Langel, A proposed model for the international reference field-1965, J. Geomagn. Geoelec., 19, $335,1967$.

Chappell, C. R., Detached plasma regions in the magnetosphere, $J$. Geophys. Res., 79, 1861, 1974.

Chappell, C. R., K. K. Harris, and G. W. Sharp, The morphology of the bulge region of the plasmasphere, J. Geophys. Res., 75, 3848, 1970.

Chen, L., and A. Hasegawa, A theory of long-period magnetic pulsations, 1, Steady state excitation of field line resonance, J. Geophys. Res., 79, 1024, 1974a.

Chen, L., and A. Hasegawa, A theory of long-period magnetic pulsations, 2, Impulse excitation of surface eigenmode, J. Geophys. Res., 79, 1033, 1974b.

Cornwall, J. M., Cyclotron instabilities and electromagnetic emission in the ultralow frequency and very low frequency ranges, $J$. Geophys. Res., 70, 61, 1965.

Cornwall, J. M., On the role of charge exchange in generating unstable waves in the ring current, J. Geophys. Res., 82, 4699, 1977.

Cornwall, J. M., F. V. Coroniti, and R. M. Thorne, Turbulent loss of ring current protons, J. Geophys. Res., 75, 4699, 1970.

Cowley, S. W. H., Pitch angle dependence of the charge-exchange lifetime of ring current ions, Planet. Space Sci., 25, 385, 1977.

Dwarkin, M. L., A. J. Zmuda, and W. E. Radford, Hydromagnetic waves at $6.25 R_{E}$ with periods between 3 and $240 \mathrm{~s}, J$. Geophys. Res., $76,3668,1971$.

Feigin, F. Z., E. T. Matveeva, and V. A. Troitskaya, High latitude observations of Pclb geomagnetic pulsations, report, Inst. of the Phys. of the Earth, Acad. of Sci., Moscow, USSR, 1978.

Fowler, R. A., B. J. Kotick, and R. D. Elliott, Polarization analysis of natural and artificially induced geomagnetic micropulsations, $J$. Geophys. Res., 72, 2871, 1967.

Gendrin, R., Pitch angle diffusion of low energy protons due to gyroresonant interaction with hydromagnetic waves, J. Atmos. Terr. Phys., 30, 1313, 1968.

Gendrin, R., Substorm aspects of magnetic pulsations, Space Sci. Rev., II, 54, 1970.

Gendrin, $R$., Waves and wave-particle interactions in the magnetosphere: A review, Space Sci. Rev., 18, 145, 1975.

Greifinger, P.., Ionospheric propagation of oblique hydromagnetic plane waves at micropulsation frequencies, J. Geophys. Res., 77. 2377, 1972.

Greifinger, C., and P. Greifinger, Wave guide propagation of micropulsations out of the plane of the geomagnetic meridian, J. Geophys. Res., 78, 4611, 1973.

Harris, K. K., and G. W. Sharp, Ogo-V ion spectrometer, IEEE Trans. Geosci. Electron., GE-7, 93, 1969.

Heacock, R. R., Midday Pc 1-2 pulsations observed at a subcleft location, J. Geophys. Res., 79, 4239, 1974.

Holzer, R. E., and J. A. Slavin, Magnetic flux transfer associated with expansions and contractions of the dayside magnetosphere, $J$. Geophys. Res., 83, 3831, 1978.

Kennel, C. F., and H. E. Petschek, Limit on stably trapped fluxes, $J$. Geophys. Res., 71, 1, 1966.

Kintner, P. M., and D. A. Gurnett, Observations of ion cyclotron waves within the plasmasphere by Hawkeye 1, J. Geophys. Res., 82, 2314, 1977.

Kivelson, M. G., Instability phenomena in detached plasma regions, J. Atmos. Terr. Phys., 38, 1115, 1976.

Kokubun, S., M. G. Kivelson, R. L. McPherron, C. T. Russell, and H. I. West, Jr., Ogo 5 observations of Pc 5 waves: Particle flux modulations, J. Geophys. Res., 82, 2774, 1977.

Lin, C. S., and G. K. Parks, Ion cyclotron instability of drifting plasma clouds, J. Geophys. Res., 81, 3919, 1976.

McPherron, R. L., The occurrence of Pcl magnetic pulsation activity at synchronous orbit (abstract), Eos Trans. AGU, 59, 356, 1978.

McPherron, R. L., C. T. Russell, and P. J. Coleman, Jr., Fluctuating magnetic fields in the magnetosphere, 2, ULF waves, Space Sci. Rev., 13, 411, 1972.

Pytte, R., R. L. McPherron, E. W. Hones, Jr., and H. I. West, Jr., Multiple-satellite studies of magnetospheric substorms: Distinction between polar magnetic substorms and convection-driven negative bays, J. Geophys. Res., 83, 663, 1978.

Rosenberg, R. L., and P. J. Coleman, Jr., Solar-cycle-dependent north-south, field configurations observed in solar wind interaction 
regions, Publ. 1804, Inst. of Geophys. and Planet. Phys., Univ. of Calif., Los Angeles, March 1978.

Roth, B., and D. Orr, Locating the Pcl generation region by a statistical analysis of ground based observations, Planet. Space Sci., 23. 993, 1975.

Russell, C. T., and R. M. Thorne, On the structure of the inner magnetosphere, Cosmic Electrodynamics, 1, 67, 1970.

Russell, C. T., R. L. McPherron, and R. K. Burton, On the cause of geomagnetic storms, J. Geophys. Res., 79, 1105, 1974.

S-300 Experimenters, Measurements of electric and magnetic wave fields and of cold plasma parameters on-board Geos-1: Preliminary results, Le Centre Nat. d'Etudes des Telecommun., Issy-les-Moulineaux, France, May 1978.

Solomon, J., Drift of particles and wave-particle interactions, in The Magnetospheres of Earth and Jupiter, edited by V. Formisano, p. 153, D. Reidel, Hingham, Mass., 1975.
Southwood, D. J., The hydromagnetic stability of the magnetospheric boundary Planet. Space Sci., 16, 587, 1968.

Stix, T. H., The Theory of Plasma Waves, McGraw-Hill, New York, 1962.

Taylor, W. W. L., and L. R. Lyons, Simultaneous equatorial observations of $1-$ to $30-\mathrm{Hz}$ waves and pitch angle distributions of ring current ions, J. Geophys. Res., 81, 6177, 1976.

Taylor, W. W. L., B. K. Parady, and L. J. Cahill, Jr., Explorer 45 observations of $1-$ to $30-\mathrm{Hz}$ magnetic fields during magnetic storms, J. Geophys. Res., 80, 1271, 1975.

Troitskaya, V. A., and A. V. Gul'elmi, Geomagnetic pulsations and diagnostics of the magnetosphere, Space Sci. Rev., 7, 689, 1967.

(Received June 15, 1978;

revised October 6, 1978;

accepted November 1, 1978.) 